\title{
Rendimiento diagnóstico de las punciones de lesiones biliopancreáticas guiadas por ultrasonido endoscópico con patólogo en sala
}

\section{Diagnostic performance of endoscopic ultrasound-guided fine needle aspiration of pancreatobiliary lesions with rapid on-site evaluation}

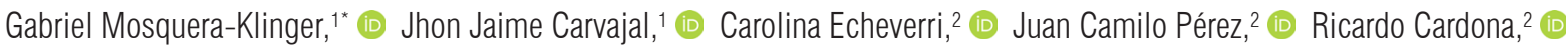
Julio Valencia-Maturana, ${ }^{3}$ (iD Héctor Sánchez-Garrido. ${ }^{1}$ (두

\author{
Gacceso abierto \\ Citación: \\ Mosquera-Klinger G, Carvajal JJ, Echeverri C, \\ Pérez JC, Cardona R, Valencia-Maturana J, \\ Sánchez-Garrido H. Rendimiento diagnóstico \\ de las punciones de lesiones biliopancreáticas \\ guiadas por ultrasonido endoscópico con \\ patólogo en sala. Rev Colomb Gastroenterol. \\ 2021;36(2):200-205. https://doi. \\ org/10.22516/25007440.628 \\ Unidad de gastroenterología y endoscopia \\ digestiva, Hospital Pablo Tobón Uribe. Medellín, \\ Colombia. \\ 2 Departamento de patología, Hospital Pablo \\ Tobón Uribe. Medellín, Colombia. \\ 3 Facultad de Medicina, Medicina Interna \\ Universidad de Antioquia. Medellín, Colombia. \\ *Correspondencia: Gabriel Mosquera-Klinger. \\ gami8203@yahoo.com \\ Fecha recibido: $\quad 27 / 07 / 20$ \\ Fecha aceptado: 18/02/2
}

\begin{abstract}
Resumen
Introducción y objetivos: el ultrasonido endoscópico con punción-aspiración con aguja fina (USE-PAAF) en lesiones neoplásicas biliopancreáticas suele tener un rendimiento alto, que depende de características de la lesión; aspectos técnicos de la USE-PAAF y la experiencia del endoscopista. De los factores menos estudiados es la presencia de patólogo en sala. Se plantea la realización de USE-PAAF con patólogo en sala para disminuir el número de pases, la tasa de muestras inadecuadas y la necesidad de repetir el procedimiento. Material y métodos: estudio observacional, retrospectivo, con recolección prospectiva de enero de 2018 a junio de 2019, en pacientes adultos sometidos a USE-PAAF. Las muestras obtenidas fueron extendidas y evaluadas en salas de endoscopia por médico patólogo con coloración Diff-Quick y cuando se obtenía una muestra suficiente se enviaba en frasco con formol para bloque celular o biopsias. Resultados: se realizaron 48 USE-PAAF biliopancreáticas en individuos con una edad mediana de 64 años. Las indicaciones más frecuentes fueron punciones por masa o pseudomasa pancreática (71 \% de casos); Se diagnosticaron 35 malignidades (77\% correspondientes a adenocarcinoma, y $14 \%$ a tumores neuroendocrinos). La mediana de tamaño de lesiones fue de $28 \mathrm{~mm}$; el número de pases promedio fue de 3 . Se obtuvieron resultados diagnósticos en $89 \%$ frente a $11 \%$ de falsos negativos. Se presentó 1 complicación menor (2,1\%), que fue dolor abdominal. Conclusiones: la USE-PAAF con patólogo en sala tiene alto rendimiento diagnóstico, con escasos resultados falsos negativos. Se requiere una mediana de pases menor, que podría minimizar los riesgos del procedimiento y la necesidad de repetir la prueba.
\end{abstract}

\section{Palabras clave}

Ultrasonido endoscópico, cáncer de páncreas, cáncer de vías biliares, punción/aspiración con aguja fina.

\begin{abstract}
Introduction: Endoscopic ultrasound with fine-needle aspiration (EUS-FNA) of pancreatobiliary neoplastic lesions usually has a high performance that depends on the characteristics of the lesion, technical aspects, and expertise of the endoscopist. One of the least studied factors is rapid on-site evaluation with a pathologist in the room. Objective: To perform EUS-FNA with a pathologist in the endoscopy room to reduce the number of passes, the rate of inadequate samples and the need to repeat the procedure. Material and methods: Observational retrospective study with a prospective data collection approach from January 2018 to June 2019 of adult patients undergoing EUS-FNA. The samples obtained were spread and evaluated in endoscopy rooms by a pathologist with Diff-Quick stain, and when a sufficient sample was obtained, it was sent in a vial with formalin for cell block and/or biopsy. Results: 48 pancreatobiliary EUS-FNA were performed in individuals with a median age of 64 years. The most frequent indication was aspiration due to pancreatic mass (71\%); 35 malignancies were diagnosed ( $77 \%$ were adenocarcinomas and $14 \%$ were neuroendocrine tumors). The median size of the lesions was $28 \mathrm{~mm}$, and the average number of passes was 3 . Diagnostic results were obtained in $89 \%$ vs. $11 \%$ of false negatives. There was 1 minor complication (2.1\%), which was abdominal pain. Conclusions: EUS-FNA with an in-room pathologist has a high diagnostic performance, with few false negative results. Also, a lower median number of passes is required, minimizing the risks of the procedure and the need for repeating it.
\end{abstract}

\section{Keywords}

Endoscopic ultrasound; Pancreatic neoplasms; Biliary tract cancer; Fine needle aspiration. 


\section{INTRODUCCIÓN}

El ultrasonido endoscópico (USE) se utiliza en el abordaje de patologías biliopancreáticas por su buena resolución para evaluar la vía biliar extrahepática y páncreas, y por la posibilidad de tomar muestras con visión directa y en tiempo real. El ultrasonido endoscópico con punciónaspiración con aguja fina (USE-PAAF) es el método de elección para toma de muestras de lesiones sólidas pancreáticas (1-3). Se considera que es más sensible que la TAC de abdomen y que la RMN en lesiones menores de $10 \mathrm{~mm}$ de tamaño. Además, es un método seguro y costoefectivo, ya que provee un alto rendimiento diagnóstico (4). El rendimiento diagnóstico se incrementa cuando se adiciona PAAF o biopsias con aguja fina (BAAF), respectivamente. La precisión diagnóstica está influenciada por factores propios de la lesión como la localización, el tamaño y el tipo de lesión estudiada; se mencionan también aspectos técnicos como el número de pases, la técnica utilizada para el muestreo (aspiración, técnica de slow pull, técnica de Fanning) y la experiencia del endoscopista; y entre los factores menos estudiados está la presencia de patólogo en sala.

Nos planteamos la realización de la USE-PAAF con patólogo en sala en todos los procedimientos para minimizar el número de pases, la tasa de muestras inadecuadas o insuficientes y la necesidad de repetir el procedimiento por resultados falsamente negativos.

\section{MATERIALES Y MÉTODOS}

Estudio observacional, retrospectivo, con recolección prospectiva de la información en pacientes mayores de 18 años sometidos a USE-PAAF en el hospital. El USEPAAF fue realizado por 2 operadores (GMK, JJC), acompañados de patólogo en sala (CE, JCP y RC). Se utilizó endosonógrafo lineal Fuji 580UT, con agujas Expect Needle o Acquire 22 G y 25 G.

Las muestras obtenidas de la USE-PAAF fueron extendidas por gastroenterólogo y patólogo; luego evaluadas inicialmente por el patólogo en salas con coloración DiffQuick, cuando se obtenía una muestra suficiente se enviaba en un frasco con formol para bloque celular o biopsias (Figuras 1A-D). La terminología utilizada para la descripción y diagnóstico final fue la clasificación citohistológica de la sociedad de Papanicolau (Bethesda).

La recopilación de las variables del estudio en la base de datos fue realizada por los coinvestigadores del estudio (JVM, HSG) luego de la revisión de historias clínicas de la institución e imágenes del sistema informático Impax ${ }^{\odot}$ o Agfa.
El protocolo fue aprobado por el comité de ética e investigaciones del hospital. Se obtuvo consentimiento informado de todos los casos antes del procedimiento.

\section{RESULTADOS}

Desde enero de 2018 hasta julio de 2019 se realizaron 48 USE-PAAF biliopancreáticas con patólogo en sala. De los 48 casos, 25 fueron hombres (52,1\%) y 23 mujeres, con edades en rangos entre 20-88 años, con una mediana de 64 años (rango intercuartílico [RIC] entre 55-71,7).

Las indicaciones para la USE-PAAF se muestran en la Tabla 1. La mayor parte de punciones se realizó en el contexto de masa en páncreas (sólida o quística) o pancreatitis crónica con pseudomasa (que constituyen el 70,83 \% de las punciones).

Tabla 1. Características clínicas y demográficas de la población

\begin{tabular}{|c|c|}
\hline Características & Valores $(n=48)$ \\
\hline \multicolumn{2}{|l|}{ Edad (RIC) } \\
\hline - Mediana de edad en años & $62,0(55,0-71,7)$ \\
\hline \multicolumn{2}{|l|}{ Distribución de edad, n (\%) } \\
\hline - Sexo masculino & $25(52,1)$ \\
\hline \multicolumn{2}{|l|}{ Clasificación ASA, n (\%) } \\
\hline - ASAII & $3(6,3)$ \\
\hline - ASA III & $44(91,7)$ \\
\hline - ASAIV & $1(2,1)$ \\
\hline \multicolumn{2}{|l|}{ Indicación del estudio, n (\%) } \\
\hline - Masa en el páncreas & $30(62,5)$ \\
\hline - Masa periampular & $4(8,3)$ \\
\hline - Masa del colédoco o biliar & $4(8,3)$ \\
\hline - Masa retroperitoneal peripancreática & $4(8,3)$ \\
\hline - Pancreatitis crónica & $2(4,2)$ \\
\hline - Cáncer renal (recidiva en lecho quirúrgico) & $1(2,1)$ \\
\hline - Insulinoma & $1(2,1)$ \\
\hline - Masa vesicular & $1(2,1)$ \\
\hline - Quiste complejo en el páncreas & $1(2,1)$ \\
\hline
\end{tabular}

ASA: American Society of Anesthesiologists.

La calidad de la muestra fue considerada suficiente en el $97,9 \%$ de los casos e insuficiente solo en 1 caso (2,1\%); el tamaño mínimo de las lesiones fue de $6 \mathrm{~mm}$ y el máximo, 
de $120 \mathrm{~mm}$, con una mediana de $28 \mathrm{~mm}$. El número de pases mínimo fue de 2 y el máximo, de 8 con una mediana de 3; la mediana de número de pases positivo para una muestra suficiente para diagnóstico o positividad fue de 3 . El diagnóstico final dado por la clasificación citohistológica de la sociedad de Papanicolau (Bethesda) estuvo en 3 grupos: el 97,9 \% de casos estuvo entre los grupos II (negativo para malignidad) y grupo VI (positivo para malignidad), y se observaron 35 casos del grupo VI (positivo para malignidad), 12 casos del grupo II (negativo para malignidad) y 1 caso del grupo I (no diagnóstico por muestra insuficiente). De los 35 casos positivos para malignidad, el diagnóstico fue cáncer de páncreas y tumor neuroendocrino pancreático en el $80 \%$ y colangiocarcinoma ductal en $3(8,5 \%)$, entre otros (ver Figura 1E-F, donde se presenta un caso en el que se observa una muestra significativa y hallazgos confirmatorios de un adenocarcnioma de páncreas). Cuando la localización de la lesión fue descrita en el páncreas (35 casos), en orden de frecuencia, la ubicación fue en la cabeza, cuerpo, uncinado y cuello (ver Tabla 2).

De las 13 punciones que no demostraron malignidad, 8 fueron no malignos en el escenario de lesiones con baja probabilidad preprueba para malignidad (pancreatitis crónica con pseudomasa inflamatoria, lesión sólido/quística del páncreas, bazo ectópico intrapancreático y lesión retroperitoneal en cercanía al páncreas) y 5 fueron resultados falsos negativos (masa en cuerpo de páncreas, lesión en colédoco, masa en el cuello del páncreas, insulinoma en el cuerpo del páncreas y un GIST extragástrico en cercanía al páncreas). De estos falsos negativos, se repitió el USE en 3 casos; es decir, se presentaron 43 resultados diagnósticos ( $89 \%)$, de los cuales 35 detectaron malignidades ( $81 \%$, frente al $19 \%$ de resultados con patología benigna). Además, se presentaron 5 casos $(11 \%)$ de falsos negativos.

Los endosonografistas describieron dificultades técnicas que limitaron la punción en 7 casos (14,6\%), que correspondieron a un aumento en la vascularización en la lesión o a su alrededor.

En cuanto a las agujas utilizadas, las punciones realizadas entre enero de 2018 y febrero de 2019 fueron con aguja fina para punción/aspiración (Expect Needle), del 1 de marzo hasta junio 2019 se realizaron con aguja fina para biopsia (Acquire Needle). Con respecto a estas últimas punciones con aguja de biopsia, se encontró que 18 de estas 20 fueron positivas para malignidad, 1 negativa cuando el diagnóstico preprueba era pancreatitis crónica con pseudomasa y 1 negativa en la que se documentó un GIST extragástrico en cercanía al páncreas. Esto corresponde a un $95 \%$ de eficiencia diagnóstica en estas últimas USE con aguja de biopsia (en la Figura 2 se muestra el número de casos relacionados con la aguja y el diámetro utilizado).
Tabla 2. Características de la muestra y localización

Características

Valores $(n=48)$

Calidad del material obtenido, $\mathrm{n}(\%)$

Suficiente

$47(97,9)$

Localización del páncreas $(n=35)$

- Cabeza

$13(37,1)$

- Cuerpo

$13(37,1)$

- Cola

$5(14,3)$

- Uncinado

$3(8,6)$

- Cuello

Localización extrapancreática $(n=13)$

- Lesiones biliares extrahepáticas 4 (31)

Lesiones periampulares

Masa retroperitoneal, peripancreática

Muestra diagnóstica, n (\%)

- Ambas

Bloque únicamente

Citología únicamente

Diagnóstico final de la sociedad de Papanicolaou, n (\%)

- Categoría I (no diagnóstico)

Categoría II (malignidad negativa)

Categoría IV (neoplasia benigna)

Categoría VI (malignidad positiva)

$35(72,9)$

Diagnóstico preciso $(n=43)$

Adenocarcinoma ductal pancreático

Adenocarcinoma biliar

$2(4,6)$

Tumor neuroendocrino

Linfoma

Plasmocitoma

Inflamación crónica y fibrosis pancreática

Bazo ectópico

GIST

Otros

$3(7)$

GIST: tumor estromal gastrointestinal. 

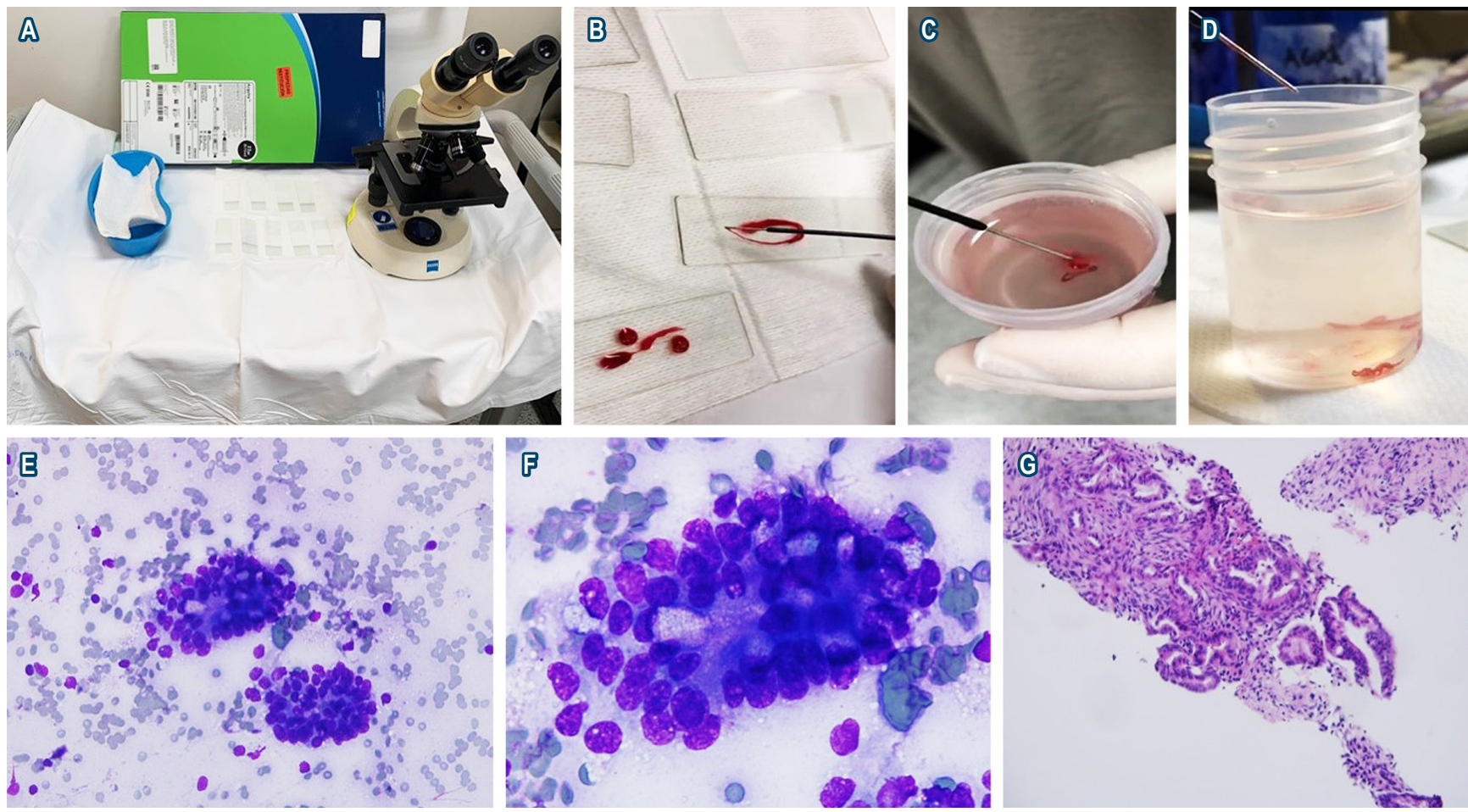

Citología 40 x Diff-Quick adenocarcinoma ductal

Citología 100 x Diff-Quick adenocarcinoma ductal

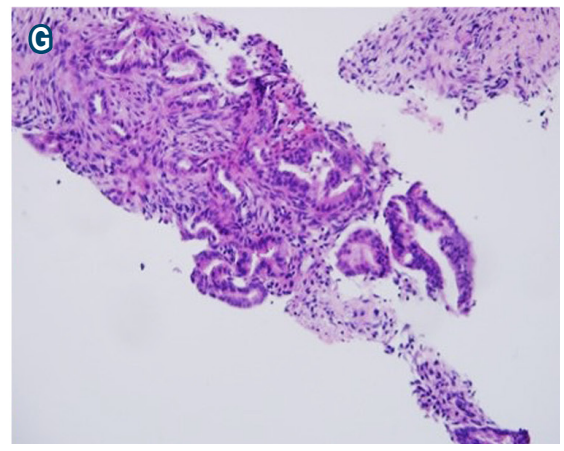

Bloque celular $40 \mathrm{XH}$ y E

Figura 1. A. Estación de trabajo con materiales utilizados por el patólogo en sala para realizar el extendido y evaluación inicial de las muestras (microscopio, láminas portaobjetos, riñonera con coloración Diff-Quick). B. Extendido de muestra obtenida en la punción en el portaobjetos. C y D. Cilindros en frasco de formol para bloque celular y biopsia. E. Citología 40 x Diff-Quick en la que se identifican 2 acúmulos de células de apariencia neoplásica. F. Citología 100 x Diff-Quick en la que se evidencia adenocarcinoma ductal. G. Bloque celular con coloración de hematoxilina-eosina (H y E) en 40x acúmulo de células neoplásicas.

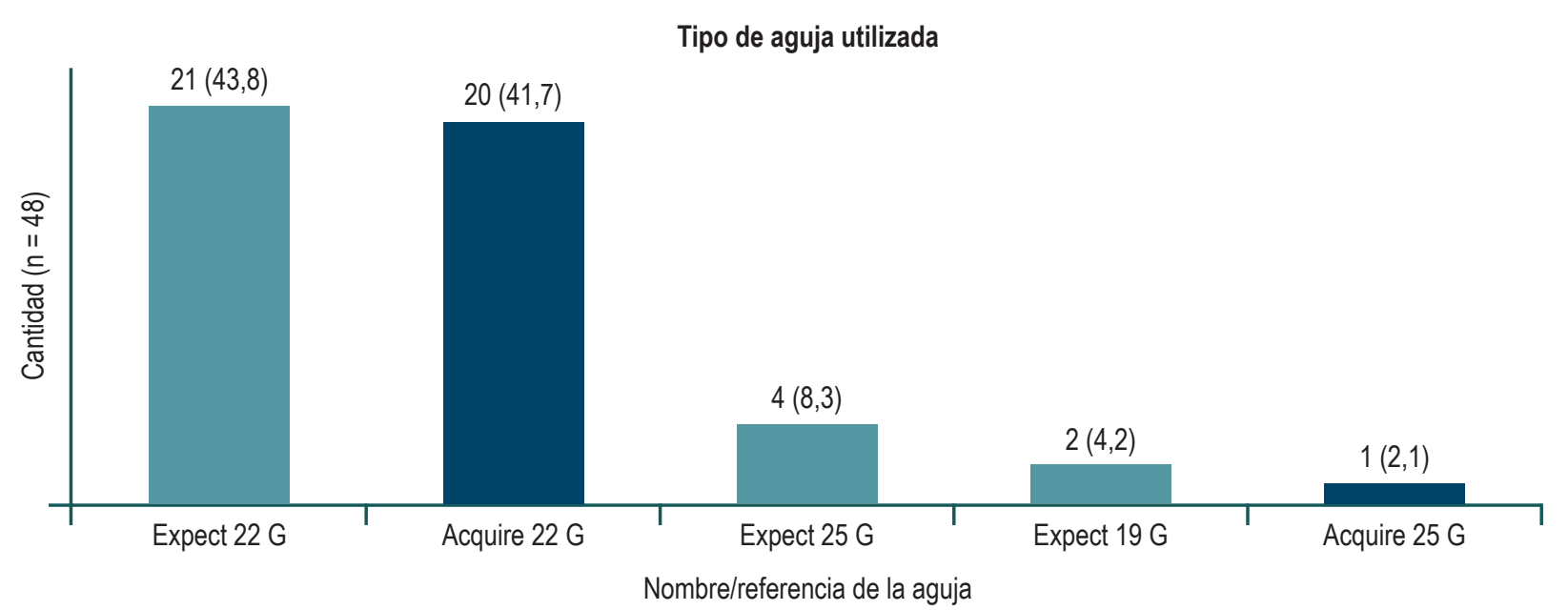

Figura 2. Distribución de tipo de aguja utilizada en las punciones.

Se presentó 1 complicación $(2,1 \%)$, que fue un dolor abdominal intenso, que requirió uso de opioides y la realización de múltiples estudios e imágenes con las que se descartó pancreatitis, perforación y sangrado.

\section{DISCUSIÓN}

En el presente estudio, el USE-PAAF con patólogo en sala tuvo un rendimiento diagnóstico alto (del $89 \%$ sin dis- 
tinción del tipo de aguja utilizada y del $95 \%$ cuando se realizó con aguja de biopsia). En la mayoría de los casos (casi en el $98 \%$ ), los patólogos consideraron muestras suficientes para establecer el diagnóstico histopatológico, con un promedio de 3 pases; nuestros datos son similares a lo descrito en el trabajo de Iglesias-García y colaboradores (5), quienes describieron una asociación significativa de la valoración citopatológica en sala con un número significativamente bajo de muestras inadecuadas y un menor número de pases de agujas (5). El número menor de pases de la aguja podría impactar en la disminución del riesgo de efectos adversos de la punción $(5,6)$. En nuestra serie solo se presentó una complicación leve (dolor abdominal), similar a lo descrito en varios trabajos grandes con grupos de alta experiencia técnica $(2,5,6)$. En nuestra serie se presentaron 5 resultados falsos negativos, que correspondieron a una masa en el cuerpo del páncreas, una lesión en el colédoco, una masa en el cuello de páncreas, un insulinoma en el cuerpo del páncreas y un GIST extragástrico en cercanía al páncreas. De estos falsos negativos se repitió el USE-PAAF en 3 casos (los cuales resultaron positivos para malignidad). Los otros 2 casos fueron correspondientes a un paciente con una lesión del colédoco, quien fue llevado a resección quirúrgica con intención curativa y en dicha patología se demostró malignidad; y a un paciente con un insulinoma en el cuerpo de páncreas sintomático al cual se le realizó ablación mediante etanolización USE-guiada. En todos los pacientes a quienes el resultado de la punción/biopsia fue positivo para malignidad, la histopatología permitió el tratamiento definitivo de los casos.

Consideramos que una de las claves para obtener mejores resultados en relación con la citología por USE-PAAF es obtener material suficiente y representativo de la lesión, y transferir adecuadamente al laboratorio de patología cuando no hay patólogo en sala. En centros de alta experiencia, con muestras obtenidas con adecuada celularidad se logra establecer el diagnóstico histológico hasta en el 75 \%-95 \% de los casos y con mínima variabilidad en la interpretación (7). En nuestro trabajo, resaltamos que la experiencia del grupo de endosonografistas es menor de 3 años, con lo cual consideramos que, a pesar de la corta experiencia del grupo se lograron los objetivos de buen rendimiento diagnóstico y de mínima necesidad de repetir procedimientos para obtener un diagnóstico preciso. En un estudio retrospectivo realizado en un centro hospitalario en Francia, se revisaron 106 casos con lesiones pancreáticas en quienes se realizó el USE-PAAF. Los resultados fueron leídos por 2 citopatólogos, quienes evaluaron la cantidad y calidad de la muestra a procesar y contaban con un tercer citopatólogo considerado experto. Las muestras inadecuadas correspondían a otro tejido o no había muestra representativa. El promedio de pases fue entre 1 y 5 con una media de 3 , igual a lo descrito en nuestro trabajo. Los resultados demostraron que la sensibilidad para detectar lesiones pancreáticas malignas mejoró con un citopatólogo experimentado ( $72 \%$ frente a $89 \%)$. Un citopatólogo experimentado reduce el número de muestras indeterminadas, aumenta el reconocimiento de lesiones malignas y proporciona una histología precisa para diagnósticos sospechosos o malignos $(8,9)$.

Por último, a pesar de que nuestro estudio no tenía como desenlace primario comparar el tipo de aguja utilizada, nuestros patólogos tuvieron una percepción de notable mejoría en la evaluación histopatológica ya que lograron realizar el estudio de los casos con mayor profundidad (inmunohistoquímica, diferenciación del tejido neoplásico, número de mitosis, entre otros), como fue descrito en el estudio de Rodrigues-Pinto y colaboradores, en el que compararon el USE-PAAF con patólogo en sala frente al USE con aguja de biopsia y se mostró que la adquisición de muestras fue técnicamente exitosa en todos los pacientes. Además, en los informes de patología describieron el tejido central de todas las lesiones BAAF con una longitud media de $15 \mathrm{~mm}$ y en cuanto a lesiones primarias neoplásicas, el rendimiento de las agujas de biopsias con respecto al diagnóstico, el grado de diferenciación, el origen metastásico, la tasa de proliferación y el diagnóstico definitivo benigno proporcionó una ventaja estadísticamente significativa $(87,9 \%$ [76 \%-100 \%] frente a $27,3 \%$ [11\%-43\%], $p<0,001)(10)$. Por lo cual consideramos que el aporte de un patólogo en sala más el USE-BAAF podría ser una estrategia a utilizar para mejorar el rendimiento, la mejor caracterización de las lesiones y, de este modo, minimizar la necesidad de repetir exploraciones y toma de muestras.

La limitación de nuestro estudio es el diseño retrospectivo. Para esta limitación e intentar disminuir sesgos de información se hizo control de la calidad del ingreso de datos con doble entrada de los mismos, aunque reconocemos que en el diligenciamiento de la historia clínica el grupo investigador no tiene control. Los datos obtenidos en nuestra cohorte son de un solo centro universitario de alta complejidad donde contamos con patólogos que pueden asistir a las punciones, por lo cual los resultados podrían no ser aplicables en todos los centros del país. Planteamos la necesidad de realizar estudios analíticos multicéntricos que comparen el rendimiento diagnóstico del USE-BAAF con y sin patólogo en sala.

\section{CONCLUSIÓN}

El USE-PAAF es un método prometedor que mejora notablemente el rendimiento diagnóstico en lesiones biliopancreáticas. La toma de muestras significativas es fundamental en un centro de excelencia biliopancreático. Esto podría 
ayudar a establecer un diagnóstico más rápido y preciso. La presencia de un patólogo en sala podría mejorar el rendimiento diagnóstico, objetivar la presencia de una adecuada muestra de tejido y requerir menor cantidad de pases de la aguja, lo que disminuye los riesgos asociados con las punciones. También podría contribuir a disminuir los tiempos en el diagnóstico por la menor necesidad de repetir los estudios, esto favorece un manejo temprano de la situación clínica y la posible reducción de costos.

\section{Agradecimientos}

A las doctoras Juliana Londoño y Leidy Tamayo por su valioso aporte en la recolección inicial de datos.

\section{REFERENCIAS}

1. Bhutani MS, Koduru P, Joshi V, Saxena P, Suzuki R, Irisawa A, et al. The role of endoscopic ultrasound in pancreatic cancer screening. Endosc Ultrasound. 2016;5(1):8-16. https://doi.org/10.4103/2303-9027.175876

2. Uehara H, Ikezawa K, Kawada N, Fukutake N, Katayama $\mathrm{K}$, Takakura R, et al. Diagnostic accuracy of endoscopic ultrasound-guided fine needle aspiration for suspected pancreatic malignancy in relation to the size of lesions. J Gastroenterol Hepatol. 2011;26(8):1256-61. https://doi.org/10.1111/j.1440-1746.2011.06747.x

3. Hewitt MJ, McPhail MJ, Possamai L, Dhar A, Vlavianos P, Monahan KJ. EUS-guided FNA for diagnosis of solid pancreatic neoplasms: a meta-analysis. Gastrointest Endosc. 2012;75(2):319-31. https://doi.org/10.1016/j.gie.2011.08.049

4. Ayres LR, Kmiotek EK, Lam E, Telford JJ. A Comparison of Endoscopic Ultrasound-Guided Fine-Needle Aspiration and Fine-Needle Biopsy in the Diagnosis of Solid Pancreatic Lesions. Can J Gastroenterol Hepatol. 2018;2018:1415062. https://doi.org/10.1155/2018/1415062

5. Iglesias-Garcia J, Dominguez-Munoz JE, Abdulkader I, Larino-Noia J, Eugenyeva E, Lozano-Leon A, et al. Influence of on-site cytopathology evaluation on the diagnostic accuracy of endoscopic ultrasound-guided fine needle aspiration (EUS-FNA) of solid pancreatic masses. Am J Gastroenterol. 2011;106(9):1705-10. https://doi.org/10.1038/ajg.2011.119
6. Schmidt RL, Walker BS, Howard K, Layfield LJ, Adler DG. Rapid on-site evaluation reduces needle passes in endoscopic ultrasound-guided fine-needle aspiration for solid pancreatic lesions: a risk-benefit analysis. Dig Dis Sci. 2013;58(11):3280-6. https://doi.org/10.1007/s10620-013-2750-6

7. Facciorusso A, Stasi E, Di Maso M, Serviddio G, Ali Hussein MS, Muscatiello N. Endoscopic ultrasound-guided fine needle aspiration of pancreatic lesions with 22 versus 25 Gauge needles: A meta-analysis. United European Gastroenterol J. 2017;5(6):846-853. https://doi.org/10.1177/2050640616680972

8. Paksoy N, Ozbek B. Cytopathologist-performed and ultrasound-guided fine needle aspiration cytology enhances diagnostic accuracy and avoids pitfalls: An overview of 20 years of personal experience with a selection of didactic cases. Cytojournal. 2018;15:8. https://doi.org/10.4103/cytojournal.cytojournal_20_17

9. Alsibai KD, Denis B, Bottlaender J, Kleinclaus I, Straub P, Fabre M. Impact of cytopathologist expert on diagnosis and treatment of pancreatic lesions in current clinical practice. A series of 106 endoscopic ultrasound-guided fine needle aspirations. Cytopathology. 2006;17(1):18-26. https://doi.org/10.1111/j.1365-2303.2006.00312.x

10. Rodrigues-Pinto E, Jalaj S, Grimm IS, Baron TH. Impact of EUS-guided fine-needle biopsy sampling with a new core needle on the need for onsite cytopathologic assessment: a preliminary study. Gastrointest Endosc. 2016;84(6):1040-1046. https://doi.org/10.1016/j.gie.2016.06.034 